\title{
Comparison of transvaginal and transabdominal ultrasonography in the diagnosis of ectopic pregnancy
}

\author{
Nahar $\mathrm{MN}^{1}$, Quddus MA ${ }^{1}$, Sattar $\mathrm{A}^{2}$, Shirin $\mathrm{M}^{3}$, Khatun $\mathrm{A}^{4}$, Ahmed $\mathrm{R}^{1}$, Sultana $\mathrm{F}^{5}$ \\ ${ }^{1}$ Department of Radiology and Imaging, Dhaka Medical College, Dhaka, ${ }^{2}$ Department of Radiology \& \\ Imaging, Sir Salimullah Medical College, Dhaka, ${ }^{3}$ Department of Radiology \& Imaging, Bangabandhu \\ Sheikh Mujib Medical University, Dhaka, ${ }^{4}$ Department of Radiology and Imaging, National Institute of \\ Cardiovascular Disease (NICVD), Dhaka, ${ }^{5}$ Department of Radiology \& Imaging, Sylhet MAG Osmani \\ Medical College, Sylhet. Email: nazmuntony@gmail.com
}

\begin{abstract}
This cross sectional study was carried out in the department of Radiology and Imaging, Dhaka Medical College Hospital from July 2008 to June 2010 to compare the accuracy of transvaginal ultrasonography and transabdominal ultrasonography in the diagnosis of clinically suspected cases of ectopic pregnancy. Initially 60 patients with clinical suspicion of ectopic pregnancy were included in this study after analyzing selection criteria 30 patients underwent both transvaginal and transabdominal ultrasonography. 'Histopathological diagnosis' was considered gold standard against which accuracies of two diagnostic modalities were compared. Sensitivity, specificity, positive predictive value, negative predictive value and accuracy of transabdominal ultrasonography as a diagnostic modality in evaluation of suspected ectopic pregnancy were $73.1 \%, 75 \%, 95 \%, 30 \%$ and $73.3 \%$ respectively where as transvaginal ultrasonography was found to have $92.3 \%$ sensitivity, $75 \%$ specificity, $96 \%$ positive predictive value, $60 \%$ negative predictive value and $90 \%$ accuracy. Transvaginal ultrasonography was superior to transabdominal ultrasonography in the evaluation of suspected ectopic pregnancies .so, transvaginal ultrasonography is important for early and accurate diagnosis of ectopic pregnancy.
\end{abstract}

\section{Introduction}

Ectopic pregnancy represents implantation of the fertilized ovum outside the uterine cavity. The incidence of ectopic pregnancy is upto 16.9 per 1000 reported pregnancies and is increasing ${ }^{1,2}$. The main problem of ectopic pregnancy is non-specific clinical presentation. Symptoms can vary from vaginal spotting to vasomotor shock with haemoperitoneum. The classic triad of short period of amenorrhoea, irregular vaginal bleeding and abdominal pain is present in $45 \%$ of patients. Both typical and atypical clinical presentation can mimic other conditions such as early pregnancy, abortion, normal intrauterine pregnancy, salpingitis, torsion or rupture of the ovarian cyst, bleeding corpus luteum, endometriosis, appendicitis, gastroenteritis, diverticulitis, diseases affecting urinary tract etc. Therefore early and reliable diagnosis of ectopic pregnancy is major challenge for clinician and is essential to avoid life-threatening bleeding or consequent infertility.

Sonography has become an important tool in the diagnosis of suspected ectopic pregnancy ${ }^{3}$. Ultrasonography is a cheap, widely available, simple, rapid and noninvasive diagnostic modality for fast detection, presence and location of pregnancy. An algorithm based on abdominal sonographic findings is suggested by authors which appears useful for the clinical evaluation of suspected ectopic pregnancies ${ }^{4}$. However, abdominal ultrasonographic accuracy can be affected by factors such as obesity, insufficient filling of bladder and obscuration of pelvic structures by bowel gas.

These problems can be reduced by the use of vaginal ultrasonography because the transducer is closer to pelvic organs than it is with the abdominal method. In addition, improved resolution may be achieved by using higher frequency transducer ${ }^{5}$. Few studies carried out abroad to compare accuracy of transabdominal \& transvaginal ultrasonography in evaluation of ectopic pregnancy but no such study yet done in our country. So, the study was carried out to see whether transvaginal ultrasonography is superior to transabdominal ultrasonography in the evaluation of the patient of suspected ectopic pregnancy by comparing the findings of the two imaging techniques with that of histopathology findings and thus to find out the sensitivity, specificity and accuracy of transvaginal ultrasonography \& transabdominal ultrasonography in the evaluation of ectopic pregnancy.

\section{Materials and Methods}

This cross sectional study was carried out in department of Radiology and Imaging, Dhaka Medical College Hospital from July 2008 to June 2010 on the patients with clinical suspicion of ectopic pregnancy. 30 patients were selected purposively meeting the following inclusion and 
exclusion criteria. Clinically suspected and incidentally detected cases of ectopic pregnancy were included in the study and those having normal or abnormal intrauterine pregnancy and those not admitted in this hospital and those with unavailability of histopathology reports were excluded. After giving a brief explanation of the procedure all underwent both transabdominal and transvaginal ultrasonography. Transabdominal sonography was performed by $3.5 \mathrm{MHz}$ probe. Transvaginal sonography was performed by 7.5 $\mathrm{MHz}$ frequency convex probe immediately after the abdominal scan with an empty bladder using standard technique. Imaging of the uterus and adnexa were performed in both sagittal and transeverse planes. During ultrasound examination, special note was made on presence of adnexal mass, peritoneal collection in Cul-De-Sac and hepatorenal pouch, extrauterine gestational sac and embryo with cardiac activity. The criteria for diagnosis of ectopic pregnancy included an extrauterine gestational sac containing a foetus or a foetal pole or an empty extrauterine sac. Solid or complex adnexal mass, peritoneal collection, peudogestational sac were considered suggestive and correlated with pregnancy test ( $\beta$ hCG in urine and serum). All patients underwent surgery and histopathology report of tissue samples collected. Ethical clearance was taken from the institutional ethical committee of Dhaka Medical College.

After collection of the data sensitivity, specificity, accuracy, positive and negative predictive values of individual transabdominal and transvaginal ultrasonography in the diagnosis of ectopic pregnancy were calculated by appropriate statistical formula. Comparison between transabdominal and transvaginal ultrasonographic findings was done by using Fisher's Exact Test. $\mathrm{P}$ value $<.05$ was considered to be significant.

\section{Results}

The diagnostic accuracy of transvaginal and transabdominal ultrasonography was compared in 30 patients with suspected ectopic pregnancy. No embryonic sac was identified within uterus in all 30 patients, who had positive pregnancy test. Using the criteria of a double decidual sign pseudosac and nonspecific internal uterine echoes were identified (fig 1). Out of 26 histopathologically proved ectopic pregnancy 24 cases were diagnosed by transvaginal ultrasonography while transabdominal ultrasonography diagnosed 19 cases.

In 5 out of 24 ectopic pregnancies identified by transvaginal ultrasonography a gestational sac with living embryo were detected outside the endometrial cavity. In other 5 patients of ectopic pregnancies diagnosed by transvaginal ultrasonography a definite gestational sac with an echogenic rim and foetal pole or embryo were detected. Other less specific diagnostic findings on transransvaginal ultrasonography in patients with documented ectopic pregnancies included: a thick walled anechoic structure with an echogenic rim representing an ectopic gestational sac in 8 patients, a complex adnexal mass in 10 patients, cul-de-sac fluid in18 patients, pseudosac in 3 patients and nonspecific echoes in 6 patients.

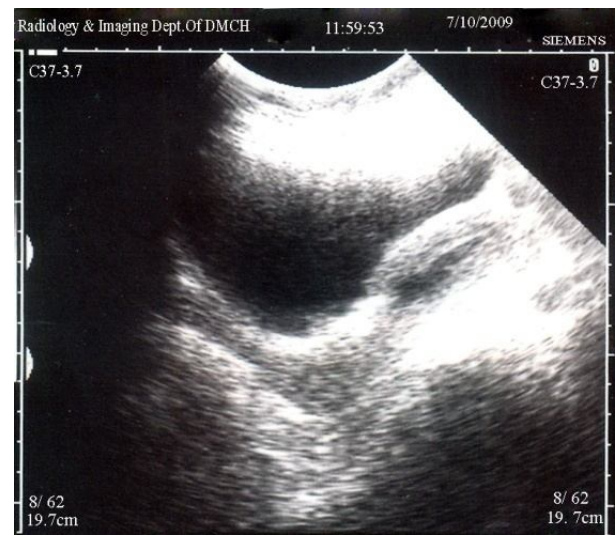

Fig.1: TAS shows sagittal view of the uterus with a debrisfilled pseudogestational sac associated with an ectopic gestation.

Transabdominal ultrasonography detected 2 cases with extrauterine gestational sac and living embryo, gestational sac with nonliving embryo in 3 cases and gestational sac without embryo or yolk sac in 5 cases. Adnexal mass was found in 8 cases and peritoneal fluid, pseudo sac and non-specific echoes in 13, 2 and 4 cases respectively. 2 ectopic pregnancies detected by transabdominal ultrasonography were missed by transvaginal ultrasonography. A gestational sac with fetus was located above uterus in one and a complex mass above the uterus was present in another patient beyond the field of view of the transvaginal probe (Fig 2).

Transvaginal ultrasonography was significantly sensitive in detecting gestational sac with or without embryo compared to transabdominal ultrasonography. Detection of adnexal mass, peritoneal fluid, pseudo sac and other nonspecific echoes were almost comparable between the two diagnostic modalities (table-I). In cases missed by transabdominal ultrasonography, transvaginal ultrasonography showed a small ectopic sac 5-12 $\mathrm{mm}$ in diameter. The ectopic sacs appeared as a ring with an echogenic contour 3-5 mm thick (Fig 3). Vaginal scanning allowed a more accurate detection of the content of the sac (fetal pole/ yolk sac) and small amount cul-de-sac fluid (Fig 4). 

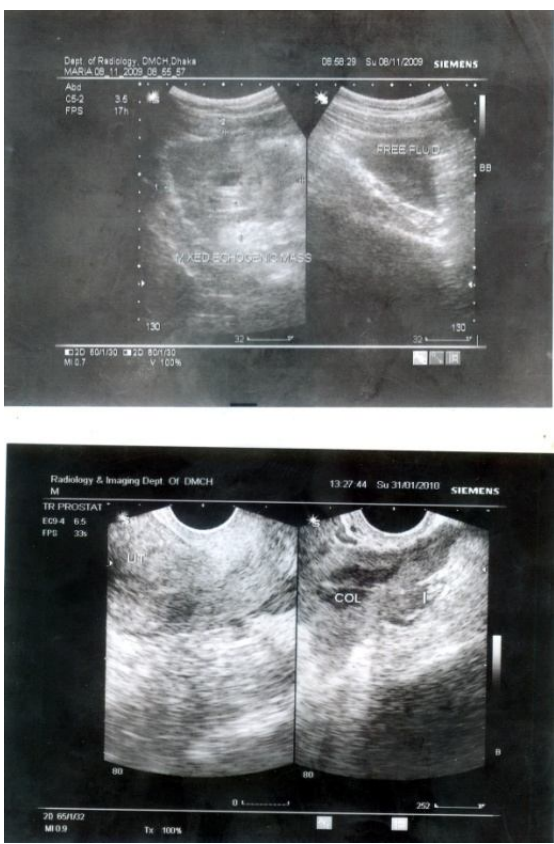

Fig. 2: Ectopic pregnancy. TVS shows empty uterus with collection in peritoneal cavity. Mixed echogenic adnexal mass in TAS missed by TVS due to its location at a higher position.

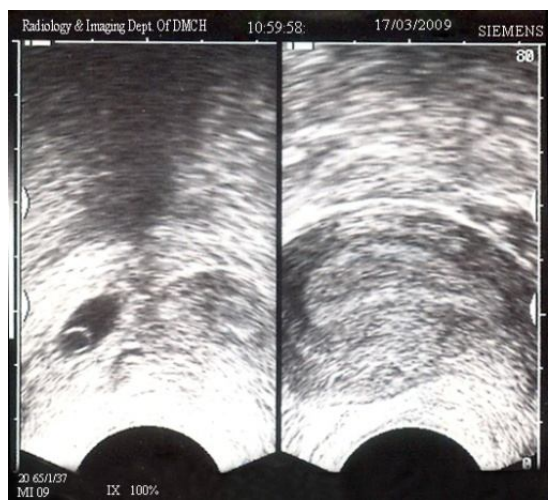

Fig. 3: TVS showing a gestational sac with foetal pole in left adnexal region. Uterus shows decidual reaction.

Out of 4 patients who had other diagnosis by histopathology 3 correlated with both diagnostic modalities and only one intrauterine pregnancy was falsely diagnosed as ectopic by each method of scanning; in this case a haemorrhagic corpus luteum cyst with abundant fluid were found at surgery.

The study result revealed that sensitivity of transabdominal and transvaginal ultrasonography was $73.1 \%$ and $92.3 \%$ respectively. Specificity of both techniques was same $(75 \%)$ may be due to small sample size. Diagnostic accuracy of transabdominal ultrasonography was $73.3 \%$ and transvaginal ultrasonography was $90 \%$. Positive predictive value and negative predictive value of transabdominal ultrasonography was $95 \%$ and $30 \%$ and of transvaginal ultrasonography $96 \%$ and $60 \%$ (Table-II \& Table-III).
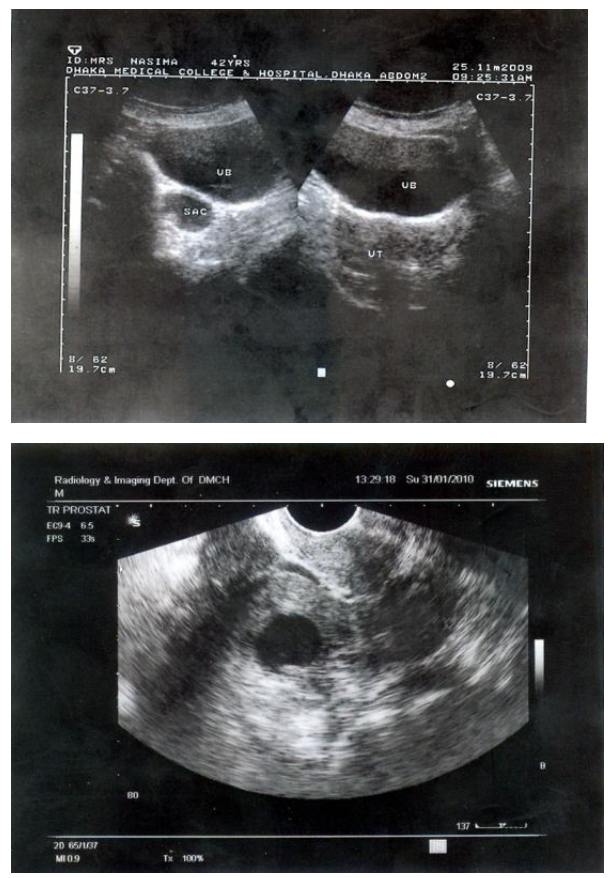

Fig. 4: Ectopic pregnancy. TAS shows empty uterus with a saclike structure in Rt. adnexa. No. internal embryo or yolk sac was demonstrated. TVS clearly demonstrates foetal pole within gestational sac.

Table I: Comparison between transvaginal and transabdominal USG Findings

\begin{tabular}{lccc}
\hline Findings & \multicolumn{2}{c}{ Diagnostic modality } & p-value \\
\cline { 2 - 3 } & $\begin{array}{c}\text { Transvaginal } \\
\text { USG (n=30) }\end{array}$ & $\begin{array}{c}\text { Transabdominal } \\
\text { USG (n=30) }\end{array}$ & \\
\hline Gestational sac & $18(60.0)$ & $10(33.3)$ & 0.035 \\
Present & $12(40.0)$ & $20(66.7)$ & \\
Absent & & & \\
Adnexal mass & $10(33.3)$ & $8(26.7)$ & 0.389 \\
Present & $20(66.07)$ & $22(73.3)$ & \\
Absent & $18(60.0)$ & $13(43.3)$ & 0.151 \\
Peritoneal fluid & $12(40.0)$ & $17(56.7)$ & \\
Present & $3(10.0)$ & $2(6.7)$ & 0.500 \\
Absent & $27(90.0)$ & $28(93.3)$ & \\
Pseudo-sac & & & \\
Present & $6(20.0)$ & $4(13.3)$ & 0.365 \\
Absent & $24(80.0)$ & $26(86.7)$ & \\
Non-specific echoes & & & \\
Present & & & \\
Absent & & & \\
\hline
\end{tabular}

Figures in the parentheses denote corresponding percentage \#Data were analyzed using Fisher's Exact Test

Table II: Accuracy of transvaginal USG in detecting ectopic

\begin{tabular}{lc}
\hline Diagnostic Accuracy & Percentage \\
\hline Sensitivity & $92.3 \%$ \\
Specificity & $75 \%$ \\
Positive Prediction Value & $96 \%$ \\
Negative Prediction Value & $60 \%$ \\
Accuracy & $90 \%$ \\
\hline
\end{tabular}

Table III: Accuracy of transabdominal USG in detecting ectopic pregnancy

\begin{tabular}{lc}
\hline Diagnostic Accuracy & Percentage \\
\hline Sensitivity & $73.1 \%$ \\
Specificity & $75 \%$ \\
Positive Prediction Value & $95 \%$ \\
Negative Prediction Value & $30 \%$ \\
Accuracy & $73.3 \%$ \\
\hline
\end{tabular}




\section{Discussion}

Sonographic evaluation of women suspected of having an ectopic pregnancy requires correct interpretation of both intrauterine and extrauterine findings. One patient may have more than one finding. Presence of intrauterine pregnancy virtually excludes an ectopic pregnancy as the incidence of concomitant intrauterine pregnancy and ectopic pregnancy is approximately 1 in 30,000. An intrauterine gestation sac with a live fetus is positive proof of an intrauterine pregnancy, but in the majority of patients the gestational age will only be 5-6 weeks and the sac will be empty. Differentiation between a true gestation sac and a pseudosac may be difficult. Using the criteria of a double decidual sign 3 pseudosac was identified by transvaginal ultrasonography, in 6 cases nonspecific internal uterine echoes were identified. Transabdominal ultrasonography detected pseudo sac and non-specific echoes in 2 and 4 cases respectively These intrauterine echoes are probably due to hyperplastic endometrium and blood within the endometrial cavity.

In the present study extrauterine gestational sac was observed in $60 \%$ cases by transvaginal ultrasonography and $33.3 \%$ cases transabdominal ultrasonography. This is in agreement with the results of Nyberg et al. ${ }^{6}$ who found ectopic sac in $65 \%$ of their patients and cacciatore et al. ${ }^{7}$ who found ectopic sac in $44 \%$ cases by transabdominal ultrasonography.

The sonographic demonstration of a live embryo in the adnexa is specific for the diagnosis of ectopic pregnancy. This finding was demonstrated in 5 (16.7\%) cases with ectopic pregnancy diagnosed by transvaginal ultrasonography. This is comparable to cacciatore et al. ${ }^{7}$ who detected ectopic foetus in $21 \%$ cases. In the present study transabdominal ultrasonography detected live embryo in $2(6.7 \%)$ cases. This is consistent with Hussain \& Haque ${ }^{8}$ who detected live embryo in $8 \%$ cases by transabdominal ultrasonography.

In other $8(26 \%)$ cases ectopic pregnancy diagnosed by transvaginal ultrasonography and 5 (16.6\%) cases by transabdominal ultrasonography a thick walled anechoic structure with an echogenic rim representing an ectopic gestational sac without any embryo/yolk sac was observed. This is not far from Thorsen et al ${ }^{6}$.

Nonspecific features like adnexal mass, peritoneal collection, and pseudogestational sac and nonspecific echoes were detected in $33.3 \%, 60 \%, 10 \%$ and $20 \%$ cases respectively by transvaginal ultrasonography. The corresponding findings were $26.7 \%, 43.3 \%, 6.7 \%$ and $13.3 \%$ in case of transabdominal ultrasonography. No significant difference noted between two modality and this correlated well with other studies, ${ }^{\mathbf{6 , 9}}$.

The major advantages of transvaginal ultrasonography were an accurate evaluation of adnexal texture and improve visualization of the contents of the sac, shown also by previous studies ${ }^{\mathbf{6 , 1 0}}$. As a result, more ectopic pregnancies were diagnosed while still unruptured.

Transvaginal ultrasonography diagnosis exhibited $25(83.3 \%)$ ectopic pregnancy and $5(16.7 \%)$ other diagnosis. Transabdominal ultrasonography diagnosis was 20(66.7\%) ectopic pregnancy, $10(33.3 \%)$ other diagnosis.

Histopathological diagnosis showed that out of 30 cases 26(86.7\%) had ectopic pregnancy and $4(13.3 \%)$ had other diseases. 24 cases were diagnosed as ectopic pregnancy by transvaginal ultrasonography. This discrepancy between transvaginal ultrasonography and histopathological diagnosis was due to 2 cases of ectopic pregnancy were falsely diagnosed as pelvic inflammatory disease by transvaginal ultrasonography.

Out of 26 histopathologically proved ectopic transabdominal ultrasonogrphic diagnosis was ectopic pregnancy in 19 cases and 7 cases were falsely diagnosed as pelvic inflammatory disease (5) and normal findings (2).

Out of 4 patients who had other diagnosis by histopathology 3 correlated with both transvaginal and transabdominal ultrasonography and 1 case of haemorrhagic corpus luteal cyst was falsely diagnosed as ectopic pregnancy by both modality.

It was evident from present study that transvaginal ultrasonography was superior to transabdominal ultrasonography. For cases in which transvaginal ultrasonography was superior, this method provided clear evidence of ectopic pregnancy in 5 cases in which transabdominal ultrasonography demonstrated nonspecific masses or normal adnexa. In other cases where both methods led to correct diagnosis, transvaginal ultrasonography provided additional useful information. In 2 cases transvaginal ultrasonography missed ectopic pregnancy that was located in a high location beyond the field of view of transvaginal transducer.

The study result revealed that sensitivity of transabdominal and transvaginal ultrasonography was $73.1 \%$ and $92.3 \%$ respectively. Specificity of both techniques was same $(75 \%)$ may be due to small sample size. Diagnostic accuracy of transabdominal ultrasonography was $73.3 \%$ and transvaginal ultrasonography was 90\%. Positive predictive value and negative predictive value of transabdominal ultrasonography was $95 \%$ and $30 \%$ and of transvaginal ultrasonography $96 \%$ and $60 \%$. 
Kim et al. ${ }^{11}$ found transabdominal ultrasonography was $95.9 \%$ sensitive and $73.9 \%$ specific. Positive predictive value was $73.9 \%$.

Hopp H. et al. ${ }^{12}$ found sensitivity of transvaginal ultrasonography $96 \%$, specificity $88 \%$, positive predictive value $95 \%$. Another study conducted by Timor-Tritsch ${ }^{13}$ sensitivity, specificity, positive predictive value and negative predictive value of transvaginal ultrasonography was $100 \%, 98.2 \%$, $98 \%$ and $100 \%$ respectively. The use of higher frequency transvaginal transducer probes improved the diagnosis of the ectopic gestation.

Conclusion: This study shows that transvaginal ultrasonography is superior to transabdominal ultrasonography for early detection of ectopic pregnancy, but to avoid misinterpretation both is required as transvaginal ultrasonography has limited field of view.

\section{References}

1. Doyle MB, DeCherney AH, Diamond MP; Epidemiology and etiology of ectopic pregnancy. Obstet Gynecol Clin North Am; 1991; 18: 1-17.

2. Stabile I, Grudzniskas JG; Ectopic pregnancy: a review of incidence,etiology and diagnostic aspects. Obstetric and gynecological survey; 1990; 45: 335-47.

3. Weckstein LN; Current perspective on ectopic pregnancy. Obstet Gynecol Surv; 1985; 40: 259-72.

4. Cacciatore B, Ylöstalo P, Stenman UH, Widholm O; Suspected ectopic pregnancy: Ultrasonic findings and hCG levels assessed by an immunofluorometric assay. Br J Obstet Gynaecol; 1988; 95: 497-502.
5. Nyberg DA, Filly RA, Duarte Filho DL, Laing FC, Mahoney BS; Abnormal pregnancy: Early diagnosis by US and serum chorionic gonadotropin levels. Radiology; 1986; 158: 393-6.

6. Nyberg DA, Mack LA, Hughes MP, Wang KY; Extrauterine findings of ectopic pregnancy at transvaginal us : Importance of echogenic fluid. Radiology; 1991; 178: 823-826.

7. Cacciatore B, Stenman UH, Ylöstalo Pं; Comparison of abdominal and vaginal sonography in suspected ectopic pregnancy. Obstet Gynecol; 1989; 73: 770-774.

8. Hussain FA, Haque M; Ultrasonographic diagnosis of ectopic pregnancy and correlation with surgical findings: A study with 81 cases. Bangladesh J. Ultrasonogr; 2002; 9(1):9-11.

9. Thorsen KM, Lawson LT, Aiman JE et al. ; Diagnosis of ectopic pregnancy: Endovaginal vs Transvaginal sonography. AJR; 1990; 155: 307-310.

10. Fleischer AC, Pennell RG, Mckee MS; Ectopic pregnancy: Features of transvaginal sonography. Radiology; 1990; 174: 375-378.

11. Kim DS, Chung SR, Park MI, Kim YP; Comparative review of diagnostic accuracy in tubal pregnancy: A 14-year survey of 1040 cases. Obstetrics and Gynaecology; 1987; 70(4):547-554.

12. Hopp H, Schaar P, Hundertmark S et al. ; Diagnostic reliability of vaginal ultrasound in ectopic pregnancy. Geburtshilfe Frauenheilkd; 1997; 55: 666-70.

13. Timor - Trisch IE, Yeh MN, Peisner DB; The use of transvaginal ultrasonography in the diagnosis of ectopic pregnancy. Obstet Gynecol; 1989; 161: 157161. 
